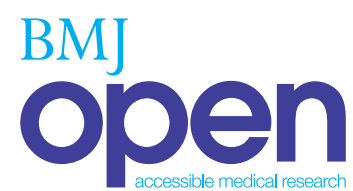

\title{
Effectiveness and cost-effectiveness of a novel, group self-management course for adults with chronic musculoskeletal pain: study protocol for a multicentre, randomised controlled trial (COPERS)
}

To cite: Carnes D,

Taylor SJC, Homer K, et al. Effectiveness and costeffectiveness of a novel, group self-management course for adults with chronic musculoskeletal pain: study protocol for a multicentre, randomised controlled trial (COPERS). BMJ Open 2013;3:e002492. doi:10.1136/bmjopen-2012002492

- Prepublication history for this paper are available online. To view these files please visit the journal online (http://dx.doi.org/10.1136/ bmjopen-2012-002492).

Received 17 December 2012 Revised 17 December 2012 Accepted 20 December 2012

This final article is available for use under the terms of the Creative Commons Attribution Non-Commercial 2.0 Licence; see http://bmjopen.bmj.com

For numbered affiliations see end of article.

Correspondence to Dr Dawn Carnes; d.carnes@qmul.ac.uk

\section{ABSTRACT}

Introduction: Chronic musculoskeletal pain is a common condition that often responds poorly to treatment. Self-management courses have been advocated as a non-drug pain management technique, although evidence for their effectiveness is equivocal. We designed and piloted a self-management course based on evidence for effectiveness for specific course components and characteristics.

Methods/analysis: COPERS (coping with persistent pain, effectiveness research into self-management) is a pragmatic randomised controlled trial testing the effectiveness and cost-effectiveness of an intensive, group, cognitive behavioural-based, theoretically informed and manualised self-management course for chronic pain patients against a control of best usual care: a pain education booklet and a relaxation CD. The course lasts for $15 \mathrm{~h}$, spread over 3 days, with a $-2 \mathrm{~h}$ follow-up session 2 weeks later. We aim to recruit 685 participants with chronic musculoskeletal pain from primary, intermediate and secondary care services in two UK regions. The study is powered to show a standardised mean difference of 0.3 in the primary outcome, pain-related disability. Secondary outcomes include generic health-related quality of life, healthcare utilisation, pain self-efficacy, coping, depression, anxiety and social engagement. Outcomes are measured at 6 and 12 months postrandomisation. Pain self-efficacy is measured at 3 months to assess whether change mediates clinical effect.

Ethics/dissemination: Ethics approval was given by Cambridgeshire Ethics 11/EE/046. This trial will provide robust data on the effectiveness and cost-effectiveness of an evidence-based, group self-management programme for chronic musculoskeletal pain. The published outcomes will help to inform future policy and practice around such self-management courses, both nationally and internationally.

Trial registration: ISRCTN24426731.

\section{ARTICLE SUMMARY}

Article focus

- We outline all procedures for a pragmatic trial investigating the effectiveness of a self-management intervention for chronic musculoskeletal pain.

Key messages

- Complex interventions need to be based on evidence and have good theoretical underpinning.

- The value of pilot studies cannot be underestimated.

- Identifying patients with non-electronically indexed conditions is difficult.

Strengths and limitations of this study

- This is a complex intervention with good theoretical underpinnings

- The trial protocol was informed comprehensive pilot study

- Difficulties surround the identification patients with non-electronically indexed conditions such as chronic pain.

\section{BACKGROUND}

Chronic conditions, especially musculoskeletal conditions, impose an increasing burden on healthcare systems and society. ${ }^{1}$ Point estimates of the prevalence of chronic musculoskeletal pain (pain lasting for longer than the normal soft tissue healing time of around 12 weeks $^{2}$ ) range from $46 \%$ to $76 \%{ }^{2}$ In 2008, the annual report on public health of the UK Chief Medical Officer identified chronic pain as a major issue which needed to be addressed. ${ }^{4}$ Despite increased understanding of the factors contributing to the development of chronic pain, ${ }^{5}$ there has been little improvement in how successfully it is treated and managed. ${ }^{6}$ Current 
treatment tends to centre on drug regimens for pain control and depression, physiotherapy and painmanagement programmes. However, many patients will experience complex care pathways involving multiple referrals to secondary care and often unnecessary and repeated diagnostic tests. ${ }^{7}$

Several UK Department of Health reports have recommended self-care for chronic disease as a strategy for improving the long-term future of health in the UK. ${ }^{8-10}$ Since 2000, the UK has invested in the implementation of lay-led (ie, peer-led) self-management training courses, the Expert Patients Programme (EPP). ${ }^{10}$ The available evidence, however, suggests that these courses may not reduce healthcare resource use as expected ${ }^{11-13}$ and that there are only modest short-term beneficial effects on other outcomes. Very few studies have examined the long-term clinical effects. ${ }^{13-15}$

In response to the anomaly between continued UK government support of self-management programmes and equivocal evidence of their effectiveness, the COPERS (coping with persistent pain, effectiveness research into self-management) study (http://blizard.qmul.ac.uk/ research-generation/329-copers.html) was commissioned by the National Institute of Health Research (NIHR), as a 5-year programme grant to identify which components and characteristics of self-management and pain-management programmes are the most effective, as well as to evaluate these components in a trial, in order to inform both the development of a new pain self-management course and the next generation of self-management courses in general. We completed two systematic reviews and a qualitative study that informed the development of the COPERS intervention. One review collated the evidence base for components and characteristics of pain and selfmanagement courses and the other collated the evidence for predictors, mediators and moderators of outcomes in these programmes. ${ }^{16}{ }^{17}$ In a qualitative study, we explored the views and experiences of participants and tutors from self-management courses about expectations, course content, recruitment, tutoring and attendance. ${ }^{18}$ The findings from this work suggested that the group-delivered courses led by healthcare professionals and/or lay people were most likely to be effective. We also found that courses lasting for a shorter time appeared to be as effective as longer courses and that the setting in which courses were delivered (ie, in healthcare settings or the community) appeared to make little difference to any beneficial effects. Evidence also suggested that participants particularly enjoyed the relaxation elements of courses but were averse to exercise being included.

Our systematic reviews found evidence to support psychological approaches; therefore, we based our course around concepts from cognitive behavioural therapy and acceptance and commitment therapy. ${ }^{19}{ }^{20}$ Some elements of the COPERS intervention are similar to the EPP, such as the inclusion of relaxation, goal-setting and action-planning and improving consultations with healthcare professionals. We also included sessions related to improving posture and social activities, for example, art and massage. Self-efficacy has been reported as an important mediator for success in these types of 'treatment' approaches. ${ }^{21}$ Since healthcare professionals and lay tutors have both been reported as effective facilitators for self-management programmes, we used both. ${ }^{22} 23$ There is mixed evidence for the optimal duration of self-management courses and 'dose' per week. Short weekly sessions over 6 weeks or more are the usual model, but attendance can be poor. ${ }^{14}$ However, evidence from mental health research shows that brief/intensive interventions can be effective and are often preferred. ${ }^{24}{ }^{25}$ The COPERS course is delivered as a 3-day intensive course on consecutive days, the rationale being to address the high attrition often reported anecdotally in other programmes. ${ }^{14}$

The protocol has been designed as a result of a feasibility study which enabled us to modify our approach to recruitment, intervention and to test a variety of outcome measures. Recruitment was problematic in the feasibility study as there is no specific electronic diagnostic code for chronic pain. We have since piloted a search strategy based on repeat prescriptions, 'Read' codes and frequency of visits, which identify many of our target population. To optimise recruitment, we decided to also use face-to-face consultations and self-referral via advertisements in surgeries and clinics. We found that filling courses within a set time period was difficult especially when, by chance, the majority of participants were randomised to the control. We have accommodated this by using randomly varied permuted block randomisation and phasing the recruitment of patients to specific time periods between school holidays.

We convened a focus group consisting of two experts in outcome measures, two general practitioners (GPs), two psychologists and two patients with chronic pain. This focus group selected the most appropriate measures to test in the pilot trial. In addition to piloting quantitative data collection, we evaluated the intervention thoroughly during the pilot study using feedback from observers, participants and facilitators. The pilot study indicated that the COPERS self-management course was viable and well received by participants, with good attendance $(85 \%$ full attendance). We found that the quality of facilitation was a key determinant for the success of a course and invested more time and resources in facilitator training and recruitment for the main trial. Additionally, we found that the group nature of the course was valued highly by participants; therefore, we allowed for more socialising during the intervention.

\section{Aims of the study}

The aim of this study is to establish the effectiveness and cost-effectiveness of the new self-management (COPERS) course for those with chronic musculoskeletal pain, when compared with usual care plus a CD recording of simple relaxation exercises (also received by the intervention arm via the self-management course). 


\section{Trial purpose}

This trial will provide the data needed by the National Health Service (NHS) purchasers to decide whether or not to fund programmes of this nature.

\section{METHODS/DESIGN}

\section{Trial design}

A multicentre pragmatic randomised controlled trial (RCT) was conducted in the UK with unbalanced randomisation (1.33: 1) (intervention:control) to accommodate for the effects of clustering. The unbalanced randomisation may also improve recruitment; if participants are told that they are more likely to receive the active treatment, then they may be more likely to join the study.

\section{Trial setting}

The trial is based in northeast London, and in the Coventry and Warwickshire region. Participants are recruited from primary and secondary care settings, such as general practices, musculoskeletal physiotherapy units and pain clinics. The population of these two localities, taken together, is broadly representative of the UK as a whole.

\section{Target population}

Inclusion criteria

- People aged 18 or over with chronic musculoskeletal pain of more than 3 months. ${ }^{2}$ This includes pain from osteoarthritis and fibromyalgia or chronic widespread pain.

\section{Exclusion criteria}

- Inability to give informed consent

- Not fluent in English

- Chronic pain arising from active malignant disease

- Pain from inflammatory arthritis, such as rheumatoid arthritis

- Serious active comorbidity or terminal illness

- Serious mental health or substance abuse issues which render the individual unable to attend the course.

\section{Participant recruitment}

Participants are recruited from primary and secondary care using three methods.

Our main method of recruitment will be bulk mail outs from targeted searches of the secondary care clinic and general practice electronic records. Electronic records are searched by identified key liaison staff in the practices and clinics. These personnel use a generic search strategy developed and tested by the study team. The search strategy has a tiered approach focusing on demographic data (patient registered at the practice, alive and $>18$ years old), repeat prescriptions for analgesics, tricyclic antidepressants and anxiolytics, followed by clinical symptoms (eg, low-back pain, back pain, osteoarthritis, fibromyalgia) and attendance in the previous 3-month period. During pilot testing, these searches identified 5-7\% of general practitioners as possibly eligible and roughly identified our target population in general practices.

The list of potential participants identified via electronic search is screened by clinicians at the practice or clinic to check suitability based on the eligibility criteria. Eligible patients are invited to join the study by letter from their GP or secondary care clinic. They are asked to complete an expression of interest form to send to the study team or to contact the study team directly. Clinicians working in participating practices are also able to invite patients consulting with chronic pain to participate by handing them an information pack. Finally, advertisements and invitation packs will be placed in general practice and clinic waiting rooms to attract interested patients, who can contact the study team directly for further information.

\section{Recruitment and informed consent}

People expressing an interest in the study, who are eligible and available to participate, are sent a baseline questionnaire, a trial consent form to complete and a freepost envelope to return the questionnaire and trial consent form to the study team. All participants are telephoned and asked whether they need more information, and the consent form is discussed before a member of the study team counter signs the consent form indicating informed consent. A summary of participant flow through the study is shown in figure 1 .

\section{Intervention group}

The intervention is a group-based, facilitated learning course supporting patients to manage their chronic pain better. It is a complex intervention using psychological approaches which have been shown to promote behaviour change of proven benefit in low back pain. ${ }^{26-28}$ The course also includes pain education, attention control, relaxation and visualisation techniques, social interaction and the opportunity to try a new activity unrelated to pain (table 1). Teaching and learning methods include facilitated learning through group discussion, sharing narratives and experiences to foster social interaction, problem solving, an educational DVD, role play, practising attention and distraction techniques and 'good' posture. We hypothesise that these group-delivered courses with plenty of breaks for social interaction will build confidence to manage chronic pain better, that is, improve self-efficacy, improve social integration and, where necessary, help re-activate participants to re-engage in social activities. ${ }^{29} 30$ The intervention consists of a short course run in 1 week over 3 days (typically Monday, Wednesday and Friday) during school hours (10:00-14:45), with a $2 \mathrm{~h}$ follow-up session 2 weeks later. The total course comprises 24 individual 'components' spread over 15-16 h. A detailed training manual for facilitators was developed, tested and refined in the 


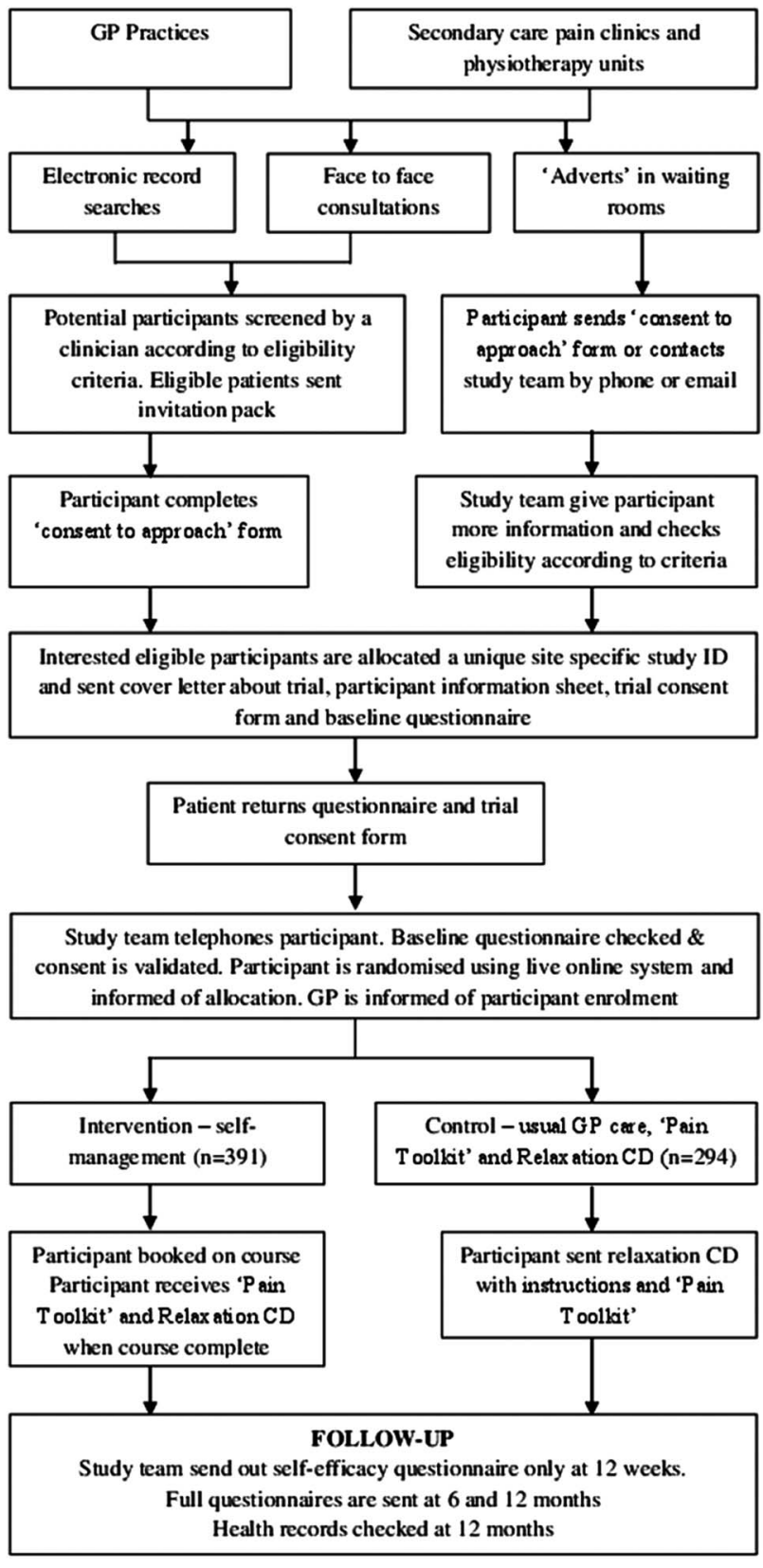

Figure 1 Flow diagram of the study.

pilot study. The manual provides the aims and theoretical basis for each component.

In the absence of any evidence favouring a particular setting for such group self-management courses, courses are held in local medical or community venues that are accessible to participants (eg, with disabled parking and near to public transport).

\section{Recruitment of facilitators for the intervention}

Each course is led by two facilitators, a healthcare professional (a professionally registered physiotherapist, osteopath, chiropractor, occupational health practitioner or psychologist) and a lay person with experience of both living with chronic pain and of tutoring or facilitating small groups.
Healthcare professional facilitators are recruited via press releases about the study in professional magazines. Lay facilitators are recruited via local contacts with community interest companies delivering self-management type programmes (typically the Expert Patient Programme, http://www.expertpatients.co.uk).

\section{Training facilitators}

Facilitators attend a 2-day training course that covers the course content, how to facilitate, dealing with difficult situations and what to do if an adverse event occurs. During the courses, the trainee facilitators are required to demonstrate actively that they are good listeners, empathic, flexible, able to encourage equal participation and laughter, able to manage difficult people, and able to introduce, lead and summarise sessions while putting the course content into a chronic pain context. We also assess their understanding of the course via a written assessment at the end of the training.

Only facilitators who are assessed as competent (ie, demonstrate the skills required during role plays and provide written answers that illustrate an understanding of the key concepts) will deliver the intervention. They are to be paired with trained and experienced facilitators from the pilot study for their first course to ensure consistency of delivery and to promote confidence in the newly trained facilitators.

\section{Control group}

In our pilot study, we found that a usual care control arm was insufficient to encourage participation in the trial. Our interviews and pilot studies suggested that participants particularly enjoyed the relaxation part of the courses, while our systematic review found little evidence that the relaxation component of pain-management courses had much observable beneficial effect. In this trial, we include a 'relaxation pack' as the control intervention to make participation more appealing. This includes a simple audio CD of three breathing and relaxation sequences, instructions and the rationale behind them. The CD is also used and distributed on the COPERS course. After randomisation, participants in the control group receive the relaxation pack and are asked to practise relaxation at least once a day every day for 3 weeks (the same duration as the intervention) and as often as they like thereafter.

In an attempt to ensure that all the study participants receive best usual care, they are also given a copy of a booklet, called the 'The Pain Toolkit', ${ }^{31}$ which is published by the UK Department of Health and freely distributed to UK GPs to give to any patient with chronic pain.

\section{Hypothesis}

We hypothesised that the benefits of the intervention will be improved function, greater self-confidence, 
Table 1 Outline of the course and theoretical models

\begin{tabular}{|c|c|c|c|}
\hline Day & Sessions & Module aims & Theory \\
\hline \multirow[t]{5}{*}{1} & $\begin{array}{l}\text { 1: Introduction } \\
\text { 2: Pain education DVD } \\
\text { 3: Acceptance: the uninvited } \\
\text { guest } \\
\text { Lunch break }\end{array}$ & Understanding pain and acceptance & $\begin{array}{l}\text { Acceptance and Commitment } \\
\text { Therapy }{ }^{19} 45\end{array}$ \\
\hline & Taster activity (eg, art) & Distract from pain perception with physical activity & Attention and distraction \\
\hline & $\begin{array}{l}\text { 4: Pain, when is it bearable } \\
\text { and when is it not? }\end{array}$ & $\begin{array}{l}\text { Pain is not just physiological; it is a psychological, } \\
\text { emotional and social phenomenon }\end{array}$ & $\begin{array}{l}\text { Biopsychosocial model of } \\
\text { medicine }\end{array}$ \\
\hline & 5: The pain cycle & $\begin{array}{l}\text { Recognising the pain cycle and signposting ways } \\
\text { out }\end{array}$ & $\begin{array}{l}\text { Fear avoidance and } \\
\text { catastrophising }^{47}\end{array}$ \\
\hline & $\begin{array}{l}\text { 6: Movement and posture } \\
\text { 7: Breathing and relaxation } \\
\text { (CD track 1) }\end{array}$ & $\begin{array}{l}\text { Reduce muscle tension to ease pain and become } \\
\text { aware of physical weakness and strengthen areas }\end{array}$ & $\begin{array}{l}\text { Physical therapy principles } \\
\text { and Alexander technique, } \\
\text { biofeedback }\end{array}$ \\
\hline \multirow[t]{7}{*}{2} & 8: Reflections from day one & $\begin{array}{l}\text { Improve social bonding, group cohesion and } \\
\text { community social support }\end{array}$ & $\begin{array}{l}\text { Social cognitive theory, } \\
\text { social learning theory }\end{array}$ \\
\hline & $\begin{array}{l}\text { 9: Identifying problems, goal } \\
\text { setting and action planning } \\
\text { 10: Barriers to change- } \\
\text { unhelpful thinking } \\
\text { Lunch break }\end{array}$ & $\begin{array}{l}\text { Recognising errors in thinking in order to promote } \\
\text { a constructive/rational view of a situation }\end{array}$ & $\begin{array}{l}\text { Cognitive therapy, } \\
\text { theories of reasoned action } \\
\text { and planned behaviour, }{ }^{50-52} \\
\text { rational emotive therapy }\end{array}$ \\
\hline & Taster activity (eg,, art) & Distract from pain perception with physical activity & Attention and distraction \\
\hline & $\begin{array}{l}\text { 11: Barriers to change- } \\
\text { reframing negatives to } \\
\text { positives }\end{array}$ & $\begin{array}{l}\text { Recognising errors in thinking in order to promote } \\
\text { a constructive/rational view of a situation }\end{array}$ & $\begin{array}{l}\text { Cognitive therapy }{ }^{2049} \text { and } \\
\text { theories of reasoned action } \\
\text { and planned behaviour }\end{array}$ \\
\hline & $\begin{array}{l}\text { 12: Attention control and } \\
\text { distraction }\end{array}$ & Distract from pain perception using visualisation & Attention control ${ }^{54}$ \\
\hline & $\begin{array}{l}\text { 13: Identifying things that } \\
\text { make pain more manageable }\end{array}$ & $\begin{array}{l}\text { Reminders to apply techniques as coping } \\
\text { strategies }\end{array}$ & Embedding learning \\
\hline & $\begin{array}{l}\text { 14: Movement and balance } \\
\text { 15: Breathing, relaxation and } \\
\text { visualisation } \\
\text { (CD track } 2 \text { ) }\end{array}$ & $\begin{array}{l}\text { Reduce muscle tension to ease pain, become } \\
\text { aware of physical weakness and strengthen areas. } \\
\text { Distraction from pain perception using visualisation }\end{array}$ & $\begin{array}{l}\text { Physical therapy principles } \\
\text { and attention management }\end{array}$ \\
\hline \multirow[t]{7}{*}{3} & 16: Reflections from day 2 & $\begin{array}{l}\text { Improve social bonding, group cohesion and } \\
\text { community social support }\end{array}$ & Social cognitive theory ${ }^{3048}$ \\
\hline & $\begin{array}{l}\text { 17: Communication with } \\
\text { health professionals } \\
\text { 18: Listening skills }\end{array}$ & $\begin{array}{l}\text { Promote constructive healthcare consultations and } \\
\text { effective communication }\end{array}$ & $\begin{array}{l}\text { Theories of reasoned action } \\
\text { and planned behaviour } \\
\text { and Social cognitive } \\
\text { theory }{ }^{30} 48\end{array}$ \\
\hline & $\begin{array}{l}\text { 19: Anger, irritability and } \\
\text { frustration }\end{array}$ & $\begin{array}{l}\text { Recognising errors in thinking in order to promote } \\
\text { a constructive/rational view of a situation }\end{array}$ & $\begin{array}{l}\text { Cognitive therapy }{ }^{20} 49 \\
\text { theories of reasoned action } \\
\text { and planned behaviour }{ }^{50-52}\end{array}$ \\
\hline & Lunch break & & \\
\hline & Taster activity (eg, art) & Distract from pain perception with physical activity & Attention management \\
\hline & $\begin{array}{l}\text { 20: Movement and stretch } \\
\text { 21: Breathing, relaxation and } \\
\text { mindfulness } \\
\text { (CD track 3) }\end{array}$ & $\begin{array}{l}\text { Reduce muscle tension to ease pain, become } \\
\text { aware of physical weakness and strengthen areas. } \\
\text { Distraction from pain perception using mindfulness }\end{array}$ & $\begin{array}{l}\text { Physical therapy principles } \\
\text { and attention management }\end{array}$ \\
\hline & 22: Summing up & $\begin{array}{l}\text { Reminders to apply techniques as coping } \\
\text { strategies }\end{array}$ & Embedding learning \\
\hline \multirow[t]{2}{*}{ Follow-up } & 23: Reflections and narratives & $\begin{array}{l}\text { Improve social bonding, group cohesion and } \\
\text { community social support }\end{array}$ & Social cognitive theory ${ }^{30} 48$ \\
\hline & 24: Managing setbacks & $\begin{array}{l}\text { Reminders to apply techniques as coping } \\
\text { strategies }\end{array}$ & Embedding learning \\
\hline
\end{tabular}

better coping skills, increased social engagement and more appropriate healthcare resource use (table 2). Our outcome measures reflect these domains.

\section{Outcomes}

For the main trial, we selected a group of measures based on both the published literature regarding their 
Table 2 Outcome measures and other data collection

\begin{tabular}{|c|c|c|}
\hline Domain & Measures & $\begin{array}{l}\text { Follow-up } \\
\text { (months)* }\end{array}$ \\
\hline Pain duration & Numerical scale & 0 \\
\hline Pain intensity & Chronic Pain Grade (pain intensity subscale) ${ }^{33}$ & $0,6,12$ \\
\hline Pain disability & Chronic Pain Grade (pain disability subscale) & $0,6,12$ \\
\hline Quality of Life & EQ-5D (health utility) ${ }^{55}$ & $0,6,12$ \\
\hline Self-efficacy & Pain Self-Efficacy Questionnaire ${ }^{56}$ & $0,3,6,12$ \\
\hline Mood & Hospital Anxiety and Depression Scale ${ }^{57}$ & $0,6,12$ \\
\hline Coping & Chronic Pain Acceptance Questionnaire ${ }^{58}$ & $0,6,12$ \\
\hline Social activity & HEIQ (Social integration subscale) ${ }^{59}$ & $0,6,12$ \\
\hline General Health & Census global health question 60 & $0,6,12$ \\
\hline Demographics & $\begin{array}{l}\text { Age, NHS number, sex, ethnicity, educational background, employment status, } \\
\text { language fluency, living arrangements (alone or with others) }\end{array}$ & 0 \\
\hline $\begin{array}{l}\text { Economic } \\
\text { analysis }\end{array}$ & $\begin{array}{l}\text { Healthcare resource use: primary care consultations, secondary care consultations, } \\
\text { hospital admissions, surgeries, imaging, tests and prescriptions from general practice } \\
\text { electronic records }\end{array}$ & 0 to 12 \\
\hline Comorbidities & $\begin{array}{l}\text { From general practice electronic records according to the Cumulative Illness Rating } \\
\text { Scale }^{7}\end{array}$ & 12 \\
\hline
\end{tabular}

validity and reliability and the extent of their use in other studies and on the focus group and pilot study.

\section{Primary outcome}

Our primary outcome is pain-related disability (we are not aiming to change pain severity in the short term, as current understanding of the mechanism of chronic pain suggests that it is unlikely to improve rapidly). ${ }^{32}$ To measure function, we chose a well-validated tool, the Chronic Pain Grade (CPG) ${ }^{33}$ The CPG is made up of two constructs-pain intensity and pain-related disability. Each construct has been validated separately. They are scored independently and can be combined to produce the Chronic Pain Grade. ${ }^{34}$ Our primary outcome is painrelated disability. This measure has three questions about pain-related function, and the responder rates their circumstance on a scale from 0 to 10 . The pain-related disability score is the mean of the three questions.

Since there is a paucity of evidence on the long-term effectiveness of these types of interventions, ${ }^{14}$ we will measure outcomes by a patient-completed postal questionnaire at baseline (before randomisation) and at 6 and 12 months after randomisation. There will be one postal reminder for each follow-up data collection point, after which non-responders will be contacted by phone to obtain the primary outcome and quality of life measures. To encourage completion and return of questionnaires, we will send participants a $£ 5$ 'high-street shop' voucher redeemable in multiple stores on a nonconditional basis along with their 6-month and 12-month questionnaires. This expression of appreciation has been shown to improve questionnaire return rates. A Cochrane review has found that 'the odds of response' were more than doubled when a monetary incentive was used (OR 2.02; 95\% CI 1.79 to 2.27) and almost doubled when incentives were not conditional on response $(1.71 ; 1.29$ to 2.26$) .^{35}$

\section{Secondary outcomes}

Table 2 shows our secondary outcomes.

\section{Criteria for withdrawal}

All participants are free to withdraw from the study at any time without having to give any explanation.

\section{Sample size}

The sample size calculation was based on detecting a standardised mean difference of 0.3 in pain-related disability between the intervention and control groups, with a power of $80 \%$ at the $5 \%$ significance level. This effect size is commensurate with the largest change seen in a recent systematic review of expert patient programmes, ${ }^{14}$ and also with the sort of change effected by interventions for other chronic pain syndromes, such as low back pain, on any continuous outcome measure. ${ }^{36}$ A simple sample size calculation indicates that we would require data on 350 subjects. We inflated the sample size because of the possibility of a 'clustering' effect in the group intervention arm and chose the ratio between intervention and control participants to increase statistical efficiency. ${ }^{37}$ Using an intracluster correlation coefficient of 0.1 and assuming, on average, 9 individuals providing data from each group resulted in 480 individuals being needed with 275 in the intervention group and 205 in the control group (1.33:1 intervention: control). Allowing, conservatively, for a $30 \%$ loss to follow-up (from an average of 13 individuals recruited per group), we sought to randomise 685 participants (391 intervention participants and 294 controls). 
Previous research and electronic record searches from the pilot study indicated that around 5\% of adults on GP registers consult with chronic musculoskeletal pain. Of these, based on our pilot study, we estimate that $10 \%$ may be interested in participating in the trial and around half of these will be recruited into the trial.

We estimated that around $80 \%$ of the population are adults over 18 years of age (www.statistics.gov.uk 2010). This means that to recruit our 685 participants, we needed a population base of around 342000 (342000 registered patients of whom approximately $80 \%$ are adults (274000). Five per cent of these may have chronic pain (13 700), and of these, we estimate that around $10 \%$ may express interest in the study (1370). Half of these may be recruited and enrolled (685). Using an average, total GP list size of 7000 , this equates to around 49 practices. We estimate that we will recruit between 12 and 14 patients per practice.

This may be an overestimate of the number of practices needed as it does not account for participants recruited from pain or musculoskeletal physiotherapy clinics or from face-to-face invitations and advertisements within general practices. After signing up to the study, general practices are given a choice of recruitment phases corresponding to blocks of prebooked course dates. Recruitment will be split roughly equally between each of our study sites.

We are aiming to book participants into courses within 12 weeks of randomisation. The minimum course size is nine, due to the cost implications of running more courses. If a course is undersubscribed $(<9)$ at the outset, it is either cancelled and those registered offered alternative dates for other courses, or those from other courses will be transferred to the more imminent courses where possible.

\section{Randomisation}

The randomisation plan was developed by the Pragmatic Clinical Trials Unit (PCTU) at Queen Mary, University of London (http://blizard.qmul.ac.uk/pragmaticclinical-trials-unit.html). Randomisation was stratified by centre (the Midlands or London). To ensure that allocation provides sufficient participants for each course, but which cannot be predicted by researchers, we used randomly varied permuted blocks of size 7 and 14 with an allocation ratio of 1.33:1 (intervention:control). Those returning completed questionnaires were telephoned by the study team. People who were able and willing to participate and gave valid informed consent were randomised over the phone. During the phone call, researchers entered the participant's trial identification number and recruiting centre into a central randomisation service, ensuring allocation concealment and participants received immediate notification of their allocation. Participants are then informed and either immediately booked on a course or sent a relaxation pack.

\section{Blinding and protection from bias}

All baseline data are collected by self-completed questionnaire prior to randomisation. After allocation, however, it is impossible to blind researchers and patients to allocation due to the nature of the intervention and the unequal randomisation. In compliance with our research ethics committee requirements, GPs are informed of their patient's enrolment into the trial but not of their allocation. Although participants are free to divulge this information to their primary care team, we feel this information in itself should have little impact on their care. Outcome data are by patient completed postal questionnaire. For non-responders, primary outcome data and the Euroqol instrument are collected over the phone by research personnel blind to treatment allocation who use a set script asking participants not to divulge their allocation prior to asking them for their data.

\section{Data management}

All data are managed in line with PCTU standard operating procedures and subject to review by audit and the Data Monitoring and Ethics Committee (DMEC). All electronic participant data are stored in encrypted and/ or password-protected files in a secure environment. A database has been designed to manage the data input to ensure consistency of practice and coding, with a built-in audit trail enabling us to track all entries and changes. Regular audit and double-checking of all primary outcomes will be conducted to ensure accuracy of data input, and a further random $10 \%$ of all data entry will be double-checked.

\section{Adverse event reporting}

These are reported via the facilitators. Minor adverse incidents (eg, a participant being tearful and distressed during a session) are logged and fed back to the study team by the end of the course. Serious adverse events related to the study (eg, extreme distress or expressing suicidal thoughts) are reported immediately to the study principal investigator in accordance with good clinical practice guidelines and, where necessary, reported to the DMEC and the study sponsor.

\section{Statistical analysis}

We will use Stata, ${ }^{37} \mathrm{R}^{38}$ and MLwiN, ${ }^{39}$ as appropriate, to analyse the data. Descriptive statistics will be used to summarise the characteristics of participants in each arm of the trial. Outcomes at follow-up will be analysed as dependent variables in mixed effects regression models. The model will include as covariates: baseline outcome measure, allocation (intervention or control), age, gender, study centre (London or the Midlands) as fixed effects, and the intervention group as a random effect. Where participants withdraw, we will compare the characteristics of those withdrawing against those who remain in the study.

Our primary analysis is an available case analysis following the intention-to-treat principles (http:// 
www.consort-statement.org/consort-statement/furtherexplanations/box6_intention-to-treat-analysis/). We will use mixed effects models appropriate for each outcome (linear, logistic, Poisson) with intervention arm, age and gender and baseline level of outcome as fixed effects and group as a random effect. The random effect will only be present in the intervention arm. We will examine patterns of 'missingness' and conduct a secondary analysis using multiple imputation and assuming variables are missing at random. We will also conduct a complier-average causal effect analysis to estimate the effect of the intervention on compliers. ${ }^{40}$ We define 'compliers' as those who attend more than half of the course (ie, those present for at least 12 of the 24 course components). We are also interested in the dose intervention participants receive, and define 'full' exposure to the intervention as being present at 17- 24 individual course sessions, moderate exposure as being present at 9 -16 components, and non-exposure as being present at eight or fewer components. We will report effect estimates with CIs. For selected outcomes, we will estimate the proportions who improve over the threshold of $30 \%$ from baseline, a figure commonly used in relation to back pain, and from this, we will estimate the numbers needed to be treated and the relative risks for improvement. ${ }^{36}$ If the analysis shows an effect of the intervention on our primary outcome (pain-related disability), we will conduct an exploratory analysis to explore whether any of the following are moderators of the effect: baseline duration of chronic pain, severity of pain-related disability and pain self-efficacy. We will also conduct a mediator analysis to determine whether the level of exposure to the intervention or a positive change in self-efficacy from baseline to 12 weeks postrandomisation mediates the effects at 6 or 12 months.

\section{Health economic analysis}

At 12 months postrandomisation, we will conduct a cost-utility analysis using EQ-5D data to measure Quality Adjusted Life years (QALYs) from an NHS perspective. The costs will cover primary care services, prescribed medication, investigations, intermediate care referrals and secondary care (using secondary user services (SUS) data). Primary care services include consultations with GPs and practice nurses, both scheduled and unscheduled. Intermediate care referrals data will be collected on physiotherapy, mental health services (including Improved Access to Psychological Therapies referrals), podiatry, community nursing, community rehabilitation and other unscheduled primary or community care (out-of-hours services and walk-in centres). SUS will include accident and emergency visits, outpatient appointments and inpatient episodes. The SUS data will have International Classification of Disease 10 and Office of Population Censuses and Surveys codes which will provide information about disease and billing data. Information derived from the Office for National Statistics death reports will be the filter for attrition by death, as the cause of death is included in this information. A secondary analysis will include broader societal costs such as the patients' out-of-pocket treatments for their pain such as complementary therapies, mobility devices, private investigations and private hospital admissions.

The data will be collected from three main sources: primary care utilisation from GP electronic records at 12 months' follow-up, SUS data from the local strategic health bodies at 15 months (since there is a 3-month 'lag' in the availability of SUS data) and patient-reported cost and utility data from the 6-month and 12-month questionnaires. Data on the costs of training sessions will be collected from trial records. Primary and intermediate care unit costs will be derived from UK published sources. ${ }^{41}$ The unit costs for medications will be obtained from the Prescription Cost Analysis database for $2010{ }^{42}$ The unit costs for secondary care will be based on health resource groups. ${ }^{43}$

If appropriate, we will use multiple imputation to guard against any bias that may result from missing EQ-5D or cost data. The imputation model will impute missing data based on age, gender, study centre (London or the Midlands), EQ-5D and cost. The imputation model for the intervention arm will also account for clustering by group in the intervention arm (by including the intervention group as a random effect).

Our primary economic analysis is a cost-utility analysis over 12 months, examining the cost per QALY gained for all participants who were assessed for EQ-5D prior to randomisation and had SUS data extracts. Descriptive statistics will be used to summarise the costs and QALYs.

We will use a mixed effects regression model to adjust estimates for costs and QALYs for the following covariates: baseline outcome measure, allocation (intervention or control), age, gender, study centre (London or the Midlands) as fixed effects, and the intervention group as a random effect. The Incremental Cost Effectiveness Ratio (ICER) will be calculated using the QALYs for each patient as a utility measurement along with the costs to the NHS incurred by each patient, where:

$$
\mathrm{ICER}=\frac{(\text { Cost of intervention }- \text { Cost of control })}{\text { Utility of intervention }- \text { Utility of control }}
$$

The spread of the incremental cost-effectiveness ratio across the four quadrants of cost effectiveness will be plotted, and we will use bootstrapping to estimate the CIs of our estimates. We also plan to assess the cost utility of the intervention using willingness to pay thresholds ranging between $£ 0$ and $£ 40000^{44}$ and to run two sensitivity analyses:

1. Excluding high-cost individuals (top 5\%).

2. Including costs from the societal perspective (out-ofpocket treatments for pain). 


\section{Quality control and fidelity of intervention delivery}

The first day of each course is observed, and where we have first time facilitators, or facilitators who lack confidence, these courses are observed in their entirety to check the fidelity of the intervention. In addition, every course is audiorecorded. A random selection of audiotaped sessions will be chosen for evaluation of fidelity. The evaluators will use a checklist to record adherence to structure and content and facilitator competence. Feedback to facilitators will be provided, where necessary, so they can modify and improve their performance.

\section{CONCLUSION}

This definitive trial will provide robust data on the effectiveness and cost-effectiveness of an optimised package of care designed to improve self-management of chronic pain. This will help to serve to inform future policy and practice for running self-management courses both nationally and internationally.

\section{Trial status}

We anticipate that our results will be complete and submitted for peer review publication in January 2014.

\author{
Author affiliations \\ ${ }^{1}$ Centre for Primary Care and Public Health, Blizard Institute Barts and The \\ London School of Medicine and Dentistry, Queen Mary University of London, \\ London, UK \\ ${ }^{2}$ Department of Psychology, Royal Holloway University of London, London, \\ UK \\ ${ }^{3}$ Department of Rheumatology, University College Hospital, London, UK \\ ${ }^{4}$ Clinical Trials Unit, Warwick Medical School, Warwick University, Coventry, \\ UK
}

Contributors MU, DC and ST conceived the original idea for the study. ST, $\mathrm{MU}, \mathrm{DC}, \mathrm{KH}, \mathrm{TP}$ and $\mathrm{AR}$ were integral to the design of the intervention and the trial, and SE, SB and MU were specifically responsible for the statistical components of the trial and the statistical analysis protocol. DC wrote the first draft of the protocol. KH, ST, MU, KH, AR, TP and SE contributed to each successive draft of the manuscript, and all authors approved the final version. $\mathrm{MU}$ and ST are joint Pls for the study; DC is the trial manager and $\mathrm{KH}$ the senior researcher; SB and SE are the trial statisticians. AR and TP are on the trial management board and provided substantial input into the intervention design.

Funding This paper presents independent research commissioned by the National Institute for Health Research (NIHR) under its Programme Grants for Applied Research scheme (RP-PG-0707-10189). The views expressed in this publication are those of the authors and not necessarily those of the NHS, the NIHR or the Department of Health.This project benefited from facilities funded through the Birmingham Science City Translational Medicine Clinical Research and Infrastructure Trials Platform, with support from Advantage West Midlands.

Competing interests None.

Ethics approval Cambridgeshire Ethics Committee provided a favourable ethical review for this study Ref: 11/EE/046. The ISCTRN is 24426731

Provenance and peer review Not commissioned; internally peer reviewed.

\section{REFERENCES}

1. Maniadakis N, Gray A. The economic burden of back pain in the UK. Pain 2000;84:95-103.

2. IASP. Pain terms a current list with definitions and notes on usage. Pain 1986;24(Suppl 1):S215-21.
3. Parsons S, Breen A, Foster NE, et al. Prevalence and comparative troublesomeness by age of musculoskeletal pain in different body locations. Fam Pract 2007;24:308-16.

4. Donaldson L. 150 years of the Annual Report of the Chief Medical Officer: on the state of public health 2008. Department of Health. Stationery Office; 2008. http://www.dh.gov.uk/en/

Publicationsandstatistics/Publications/AnnualReports/DH_096206 (accessed 17 Jan 2013).

5. Woolf CJ, Salter MW. Neuronal plasticity: increasing the gain in pain Science 2000;288:1765-9.

6. Croft P. Is life becoming more of a pain? BMJ 2000;320:1552-3.

7. Hudon C, Fortin M, Vanasse A. Cumulative Illness Rating Scale was a reliable and valid index in a family practice context. J Clin Epidemiol 2005;58:603-8.

8. Darzi A. High quality care for all: NHS Next Stage Review final report. Department of Health, Stationery Office; 2008. http://www.dh. gov.uk/en/Publicationsandstatistics/Publications/Publications PolicyAndGuidance/DH_085825 (accessed 17 Jan 2013).

9. Wanless $D$. Securing our future health: taking a long-term view-the Wanless Report. Department of Health, Stationery Office; 2002. http://webarchive.nationalarchives.gov.uk/+/http://www.hm-treasury gov.uk/consult_wanless_final.htm (accessed 17 Jan 2013).

10. Donaldson L. Expert patients usher in a new era of opportunity for the NHS. BMJ 2003;326:1279-80.

11. Department of Health. The expert patient: a new approach to chronic disease management for the 21st century. Department of Health Stationery Office; 2001. http://www.dh.gov.uk/en/Publicationsand statistics/Publications/PublicationsPolicyandGuidance/DH_4006801 (accessed 17 Jan 2013).

12. Expert patient programme. http://www.expertpatients.co.uk/ (accessed 17 Jan 2013).

13. Griffiths C, Foster G, Ramsay J, et al. How effective are expert patient (lay led) education programmes for chronic disease? BMJ 2007;334:1254-6.

14. Foster G, Taylor SJC, Eldridge SE, et al. Self-management education programmes by lay leaders for people with chronic conditions. Cochrane Database Syst Rev 2007;(4):CD005108.

15. Kennedy A, Reeves D, Bower P, et al. The effectiveness and cost effectiveness of a national lay-led self care support programme for patients with long-term conditions: a pragmatic randomised controlled trial. J Epidemiol Community Health 2007;61:254-61.

16. Miles $\mathrm{CL}$, Pincus $\mathrm{T}$, Carnes $\mathrm{D}$, et al. Can we identify how programmes aimed at promoting self-management in musculoskeletal pain work and who benefits? A systematic review of sub-group analysis within RCTs. Eur J Pain 2011;15:775.e1-775.e11.

17. Carnes D, Homer KE, Miles CL, et al. Effective delivery styles and content for self-management interventions for chronic musculoskeletal pain: a systematic literature review. Clin J Pain 2012;28:344-54.

18. Carnes D, Homer KE, Underwood M, et al. Self-management courses for those with chronic musculoskeletal pain: who to refer and why? Eur J Pain Supplements 2011;5:70-1.

19. Hayes SC, Strosahl KD, Wilson KG. Acceptance and commitment therapy: an experiential approach to behavior change. New York, USA: Guildford Press, 2004.

20. Beck JS. Cognitive therapy. New York, USA: The Guildford Press, 1995.

21. Osborne RH, Elsworth GR, Whitfield K. The Health Education Impact Questionnaire (heiQ): an outcomes and evaluation measure for patient education and self-management interventions for people with chronic conditions. Patient Educ Couns 2007;66:192-201.

22. Fransen M, McConnell S. Exercise for osteoarthritis of the knee. Cochrane Database Syst Rev 2008(4):CD004376.

23. Hayden JA, van Tulder MW, Tomlinson G. Systematic review: strategies for using exercise therapy to improve outcomes in chronic low back pain. Ann Intern Med 2005;142:776-85.

24. Watt MC, Stewart SH, Lefaivre MJ, et al. A brief cognitive-behavioral approach to reducing anxiety sensitivity decreases pain-related anxiety. Cogn Behav Ther 2006;35:248-56.

25. Wiseman CV, Sunday SR, Klapper F, et al. Short-term group CBT versus psycho-education on an inpatient eating disorder unit. Eat Disord J Treat Prev 2002:10:313-20.

26. Lamb SE, Lall R, Hansen Z, et al. A multicentred randomised controlled trial of a primary care-based cognitive behavioural programme for low back pain. The Back Skills Training (BeST) trial. Health Technol Assess 2010;14:1-253, iii-iv.

27. Henschke N, Ostelo RW, van Tulder MW, et al. Behavioural treatment for chronic low-back pain. Cochrane Database Syst Rev 2010(7):CD002014

28. Hoffman BM, Papas RK, Chatkoff DK, et al. Meta-analysis of psychological interventions for chronic low back pain. Health Psychol 2007;26:1-10. 
29. Millar NE, Dollard J. Social learning and imitation. New Haven, Connecticut, USA: Yale University Press, 1941.

30. Bandura A. Self-efficacy: toward a unifying theory of behavioral change. Psychol Rev 1977;84:191-215.

31. Moore P, Cole F. The Pain Toolkit. 2009. http://www.paintoolkit.org/ assets/downloads/Pain-Toolkit-Booklet-Nov-2012.pdf (accessed 17 Jan 2013).

32. Gwilym SE, Pollard TC, Carr AJ. Understanding pain in osteoarthritis. J Bone Joint Surg Br 2008;90:280-7.

33. Von Korff M, Ormel J, Keefe FJ, et al. Grading the severity of chronic pain. Pain 1992;50:133-49.

34. Smith BH, Penny KI, Purves AM, et al. The Chronic Pain Grade questionnaire: validation and reliability in postal research. Pain 1997;71:141-7.

35. Edwards $\mathrm{P}$, Roberts I, Clarke $\mathrm{M}$, et al. Increasing response rates to postal questionnaires: systematic review. BMJ 2002;324:1183.

36. Ostelo RW, Deyo RA, Stratford P, et al. Interpreting change scores for pain and functional status in low back pain: towards international consensus regarding minimal important change. Spine 2008;33:90-4.

37. StataCorp. Stata Statistical Software. Release 10.1. In. College Station, TX: StataCorp LP, 2007.

38. R Development Core Team. R: A Language and Environment for Statistical Computing. In.: R Foundation for Statistical Computing, Vienna, Austria, 2011.

39. Rasbash J, Charlton C, Browne WJ, et al. MLwiN Version 2.02. In: Centre for Multilevel Modelling, University of Bristol, 2005.

40. Dunn G, Maracy M, Dowrick C, et al. Estimating psychological treatment effects from a randomised controlled trial with both non-compliance and loss to follow-up. Br J Psychiatry 2003;183:323-31.

41. Curtis L. Unit Costs of Health and Social Care 2010. Personal Social Services Research Unit (PSSRU), University of Kent, 2010. https:// www.education.gov.uk/publications/eOrderingDownload/ PSSRU-1368-230X.pdf (accessed 17 Jan 2013).

42. Prescription Cost Analysis, England-2010. The Health and Social Care Information Centre, 2011. http://www.ic.nhs.uk/pubs/ prescostanalysis2010 (accessed 17 Jan 2013).

43. Department of Health: 2010-11 reference costs publication. 2011. http://www.dh.gov.uk/en/Publicationsandstatistics/Publications/ PublicationsPolicyAndGuidance/DH_131140 (accessed 17 Jan 2013).

44. Towse A. Should NICE's threshold range for cost per QALY be raised? Yes. BMJ 2009;338:b181.
45. Hayes SC, Smith SX. Get out of your mind and into your life: the new acceptance and commitment therapy. Oakland, California, USA New Harbinger Publications, 2005.

46. Turk DC, Monarch ES. Psychological approaches to pain management: a practitioner's handbook. 2nd edn. New York, USA: The Guilford Press, 2002.

47. Vlaeyen JW, Linton SJ. Fear-avoidance and its consequences in chronic musculoskeletal pain: a state of the art. Pain 2000;85:317-32.

48. Bandura A. Social Foundations of thought and action: a social cognitive theory pearson education. 1986

49. Willson R, Branch R. Cognitive behavioural therapy for dummies. Chichester, England, UK: John Wiley \& Sons, 2006.

50. Ajzen I, Fishbein M. Belief, attitude, intention and behaviour: an introduction to theory and research (Addison-Wesley series in social psychology). Harlow, Essex, UK: Longman Higher Education, 1976.

51. Ajzen I, Fishbein M. Understanding Attitudes and Predicting Social Behavior Pearson Education. Harlow, Essex, UK, 1980.

52. Kuhl J, Beckman J. eds. Action control: from cognition to behavior (springer series in social psychology). Berlin, Germany: Springer-Verlag Berlin and Heidelberg GmbH \& Co., 1985.

53. Ellis A, Dryden W. The practice of rational emotive behavior therapy. 2nd edn. New York, USA: Springer Publishing, 2007.

54. Morley S, Shapiro DA, Biggs J. Developing a treatment manual for attention management in chronic pain. Cogn Behav Ther 2004;33:1-11.

55. EuroQol Group. The EuroQol instrument:EQ-5D. 1990. http://www. euroqol.org/eq-5d (accessed 17 Jan 2013).

56. Nicholas MK. The pain self-efficacy questionnaire: taking pain into account. Eur J Pain 2007;11:153-63.

57. Zigmond AS, Snaith RP. The hospital anxiety and depression scale. Acta Psychiatr Scand 1983;67:361-70.

58. McCracken LM, Vowles KE, Eccleston C. Acceptance of chronic pain: component analysis and a revised assessment method. Pain 2004;107:159-66.

59. University of Melbourne. Health Education Impact Questionnaire. http://www.crd.unimelb.edu.au/heiq/ (accessed 17 Jan 2013)

60. Office for National Statistics. Census 2011. 2011. http://www.ons.gov.uk/census/2011-census (accessed 17 Jan 2013). 